\title{
Die Entstehung fossilisationsfähiger Schalen-Fraßreste, dargestellt am Nahrungserwerb von Homarus gammarus (Crustacea, Decapoda)
}

\author{
R. Hollmann \\ Geologisch-Paläontologisches Institut der Universität Münster, \\ Münster/Westfalen
}

\begin{abstract}
The formation of shell feeding-fragments, capable of fossilization, demonstrated on the basis of food uptake in Homarus gammarus (Crustacea, Decapoda). This paper deals with the complex behaviour of Homarus gammarus attacking the pelecypod Mytilus edulis and the gastropod Buccinum undatum; both molluscs inhabit the same nearshore environment as $H$. gammarus. The molluses were chosen from a variety of prey animals of H. gammarus in order to demonstrate formation of characteristic shell-fragments recognized by paleozoologists as traces of the meals of littoral decapods. Significant structural details of the shell-fragments are illustrated. Other shell-fragments produced by preying decapods, which often gnaw the hard shells after the meal, are not so easy to identify, although the behaviour patterns of littoral decapod crustaceans are generally quite similar.
\end{abstract}

\section{EINLEITUNG}

Uber muschel-knackende Fische und räuberische, dekapode Krebse stellte WaLTHER (1910) erste Untersuchungen in Neapel an und konnte im Aquarium nachweisen, daß Palinurus vulgaris in einem spezifischen Lebensraum große Mengen von Schalen-Bruchschill oder "Muschelsand“ zu erzeugen vermag. Weitere Angaben über Palinurus vulgaris sammelte SCHÄFER (1962, p. 146, pp. 459-462, Abb. 237-239) und erweiterte damit Beobachtungen von PAPP et al. (1947) sowie von Krejci-Graf (1926). Mit verschiedenen Bruchschill-Typen sowie mit Krebs-Fraßresten, die auf Mollusken-Nahrung zurückgehen, beschäftigten sich WiLson (1967, p. 347, Taf. 3, Fig. 1), CarTer (1967, p. 42, Taf. 1, Fig. 1-2) und CADÉE (1968, p. 84, Abb. 85). Uber das gattungs-spezifische Verhalten von Calappa beim Aufbrechen der festen Körperhüllen von Beutestücken teilt SHoup (1968) Einzelheiten aus funktionsmorphologischer Sicht mit. Zahlreiche Angaben betreffen zudem Crustaceen, die als Räuber in Austernbeeten oder als Schädlinge gegenüber anderen, der menschlichen Ernährung dienenden Mollusken gefürchtet sind (z. B. Brock 1926, Yonge 1960, Schmitt 1965).

In der mahlenden Brandung der Meeresküsten zeichnet der Feinbau der Molluskenschalen und -gehäuse die Formen der mechanisch entstandenen Bruchstücke vor: Grobe Schalenschille können danach genetisch eingeordnet werden (HoLLMANN 1968). Weitere 
Unterscheidungen der tierischen Fraßreste von anorganisch-mechanisch, rein durch Bruch gebildeten Muschelschillen, die allein von der Morphologie ausgehen, gewinnen bei der Deutung fossiler Vorkommen zusätzliche Anhaltspunkte aus der Beschaffenheit des Einbettungssedimentes und der Begleitfauna sowie deren Erhaltung. Da zudem Fraßreste das Endergebnis eines gleichbleibenden Verhaltensschemas aus einer Folge festgelegter Reaktionen darstellen, sollten an ihnen morphologische Obereinstimmungen erkennbar sein. Durch den Schalenfeinbau bedingte Bruchlinien und damit bruchschillähnliche Schalenfragmente können auch unter den Fraßresten auftreten, sind jedoch weder häufig noch typisch.

Die im Litoral verbreiteten dekapoden Krebse eignen sich als Allesfresser speziell für derartige Untersuchungen. Ihre Leistungen beim Nahrungserwerb und die dabei gebildeten Fraßreste sind im dicht- und vielschichtig besiedelten Flachwasser einfach zu untersuchen. Bevorzugt duinnschalige, juvenile und noch lebende Mollusken werden aufgebrochen, das Fleisch wird aus dem geöffneten Gehäuse herausgezupft und im Kaumagen weiter zerkleinert. Dabei werden Scheren, Extremitäten und Mundwerkzeuge brechend, zerrend und zerreißend eingesetzt. Von diesen dünnschaligen Beutetieren, die einen beträchtlichen Anteil in der Nahrung liefern, und deren Fraßresten, die gewohnheitsmäßig willkürlich über den Meeresboden verstreut werden, ist kaum etwas fossilisationsfähig.

In Anpassung an die Unwelt treten jedoch auch differenziertere Verhaltensweisen auf (SCHÄFER 1954), bei denen speziell mit einer morphologischen Umgestaltung der Greiforgane möglichst viele der wechselvollen Gegebenheiten in das Nahrungsspektrum einbezogen werden. Dabei kann das im Grunde recht unspezifische Verhalten - umweltbedingt - zu komplizierten Verhaltensmustern zusammengesetzt werden. Ein Beispiel dafür liefert Cancer pagurus, der im Versuch eine Glasröhre wie eine harte Ensis-Schale aufbricht (SCF̈̈̈ER 1954, p. 51, Abb. 116). Die diesem Brachyuren typischen Verhaltensformen beim Beuteerwerb werden auch an Attrappen in fixierter Reihenfolge nacheinander „erprobt“. Eine derartig festgelegte Reaktionsfolge kann unter Umständen dazu führen, daß außerhalb des artspezifischen Verhaltens liegende Nahrungsquellen nicht erreicht werden und daher ungenutzt bleiben. Durch die Bindung an festliegende Umweltbedingungen, einen mehr oder weniger abgegrenzten Beutebezirk sowie durch das angeborene, schematisch fixierte Verhalten bei der Beutewahl aus einem vorgegebenen, mehr oder weniger schmalen Beutespektrum werden die Möglichkeiten, die den

\footnotetext{
Abb. 1: a Derbe Tuberkeln auf dem Propodus einer Makrochela von Homarus gammarus, deren konvexe Kauflächen durch steten Gebrauch weit über die Hälfte weiß abgeschliffen sind. Die Basis der Tuberkeln ist durch äußere Chitinlamellen rötlich gefärbt (Körperlänge des Hummers $37 \mathrm{~cm}$; Zoologischer Garten Münster). $b$ Klappe von Mytilus edulis mit ausgeprägten Schürf- und Schrämmspuren, wie sie durch wiederholtes Abgleiten aus der Makrochela entstehen. $c$ Das dunkle Periostrakum ist zum großen Teil abgescheuert. In der Wirbelregion liegt die weiße, blättrige Perlmuttschicht frei. Klappe von Mytilus edulis mit schartigen Einbrüchen am distalen, dünnschaligen Außenrand: Anzeichen der einsetzenden Schalenzerstörung. $d$, $e$ Klappenreste von Mytilus edulis, deren proximale und distale Ränder abgenag sind. Durch fortgesetztes Abraspeln bereits aufgeknackter Muscheln werden weitere Schalensplitter ausgebrochen und zur Deckung des Kalkbedarfs verschluckt. Die Fraßreste erhalten dadurch typische Formen. Aufnahmen: H. Richter, Geologisch-Paläontologisches Institut Münster
} 

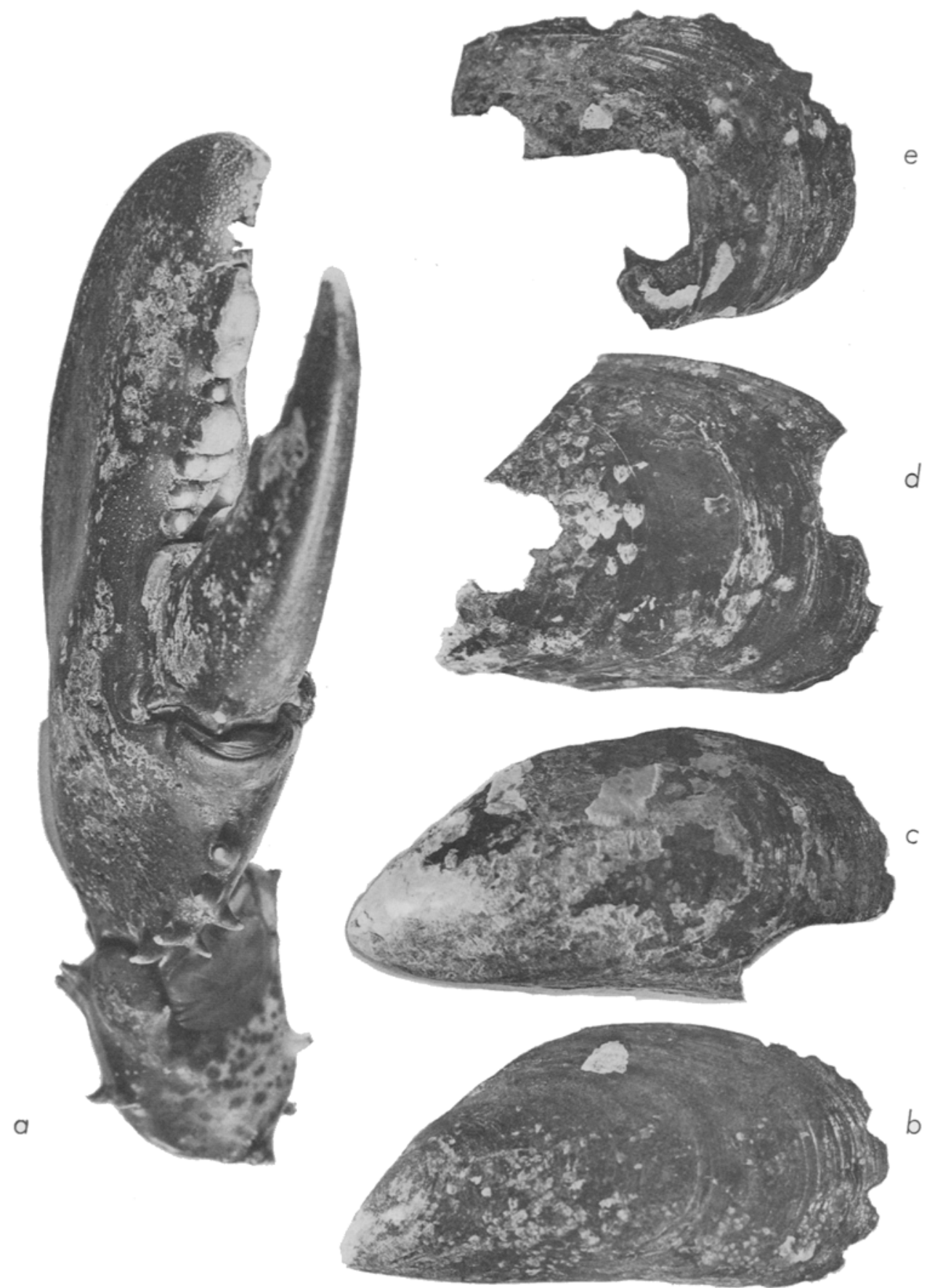
räuberischen Dekapoden im Litoral offenstehen, hingegen eingeschränkt (SCHÄFER 1962, p. 460) und damit auch die Verbreitung der artspezifischen Futterreste. Die Lernfähigkeit ist bei Dekapoden - speziell im niedrigstehenden Funktionskreis der Nahrungssuche - bis zu einem gewissen Grade möglich und kann mit zunehmender Organisationshöhe noch eine Steigerung erfahren, wodurch andersartige Futterquellen erschlossen werden (Schöne 1961, p. 510, Altevogt 1963, p. 448).

Zur Klärung derartiger Fragen wurde das Verhalten von Homarus gammarus (L.) im Aquarium gegenüber Lamellibranchiaten, Mytilus edulis (L.), und Gastropoden, Buccinum undatum L, beobachtet. Obwohl das Verhaltensschema im Aquarium adaptiv in gewissem Umfang modifiziert sein könnte, bieten sich sowohl in der Reaktionsfolge des typischen Beuteverhaltens als auch in den kennzeichnenden Formen der hartschaligen Nahrungsreste allgemeingïltige Regeln.

Diese erhalten dadurch paläontologische Bedeutung, daß die überlieferte Fossilreste Rückschlüsse auf den Lebensraum und damit auf die Meerestiefe gestatten. Zudem verdichten sich fossile Einzelbefunde zu paläoökologischen Aussagen dann, wenn die kennzeichnenden Restformen der erbeuteten Tiere auch Hinweise auf die Räuber, deren Leistungen beim Ergreifen der Beute und beim Zerstören ihrer Körperhülle erlauben. Einschränkend gilt, daß sich das Beuteverhalten bei vielen durophagen Tieren abwandeln kann und daß gleichzeitig konvergente, erhaltungsfähige tierische Restformen von verschiedenartigen Räubern geliefert werden (TEICHERT \& SERVENTY 1947, SCHärER 1962, p. 462). Manche Zuordnung mag zudem infolge Über-oder Umprägungen durch Frachtungskräfte im strömenden Wasser erschwert werden.

\section{ERGEBNISSE}

\section{Aufbrechen eines Lamellibranchiaten, Mytilus edulis}

Homarus gammarus hält sich auch im großen Seewasser-Becken biotop-getreu in Gesteinshöhlen auf. Sein Beutezug setzt stets mit einem lebhaften Spiel der 2. Antennen ein, die der Orientierung im Raum dienen und wird von differenzierten Bewegungen der Antennulae begleitet. Während das scherentragende, mit Sinnesborsten besetzte 2. und 3. Schreitbeinpaar suchend den sandigen Boden überstreicht und ihn tief aufpflügt, werden die Bewegungen der 2. Antennen und der Antennulae stetig fortgeführt. Diese stehen im Dienst der Perzeption chemischer Reize und damit dem Aufspüren und der Lokalisation der Nahrungsquellen. Beim Ergreifen der Beute oder auch anderer Gegenstände, etwa im Zusammenhang mit einer Tarnung oder mit einer Verkleinerung des Höbleneinganges, spielen die reich mit Sinnesborsten besetzten, distalen Partien der 3. Maxillen sowie das 2, und 3. Schreitbeinpaar eine wesentliche Rolle. Das Beuteobjekt wird mit den Extremitäten gepackt und dabei taktil nach Form und Struktur untersucht. Zumeist wechselt der Hummer in dieser Phase seinen Standort: Dabei hält er das erbeutete Objekt mit den im Bogen nach unten abgewinkelten 3. Maxillen allein oder zusätzlich mit dem 2. Schreitbeinpaar fest bzw. preßt es gegen den vorderen Cephalothorax. Nach erneuten, der Orientierung dienenden Dreh- und Wendebewegungen des Hummers gelangt das erbeutete Mollusk schließlich in eine günstige 


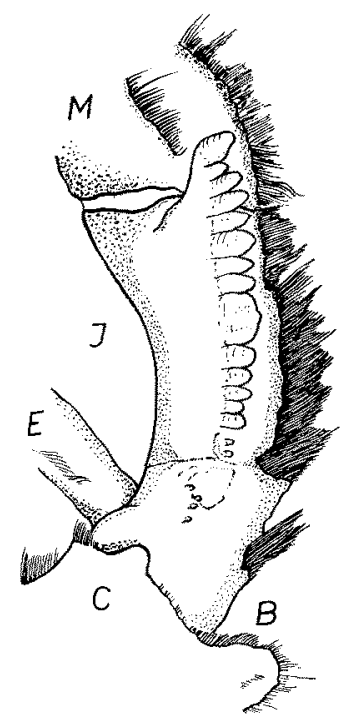

Abb. 2: Auf der Innenseite der miteinander verwachsenen Ischium-Basale erheben sich auf dem Endopoditen der 3. Maxille des Hummers eine Reihe schmaler, hoher Zähnchen. Durch den Gebrauch als bevorzugtes Halteorgan sind die rötlichen Zähnchen bis über die Hälfte wei $B$ abgeschliffen. $\mathrm{B}=$ Basale, $\mathrm{C}=$ Coxale, $\mathrm{E}=$ Exopodit, $\mathrm{I}=$ Ischium, $\mathrm{M}=$ Merus

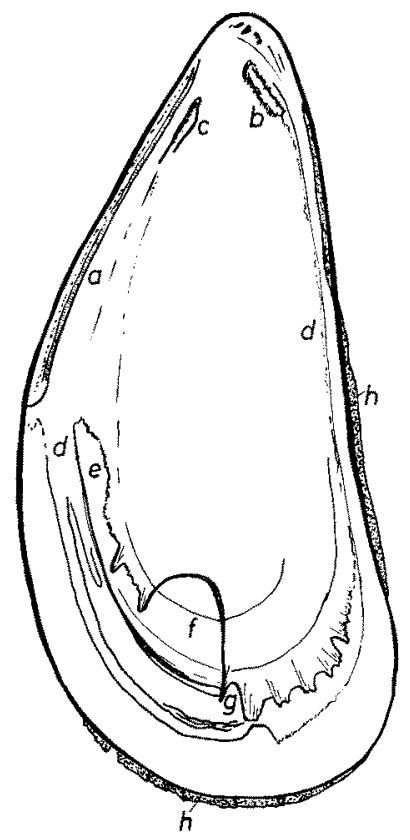

Abb. 3: Innenseite einer linken Klappe von Mytilus edulis. a Schloßband. $b$ Vorderer Schließmuskel-Ansatz. $c$ Vorderer Ansatz des Fuß-Retraktors. $d$ Muskel-Insertion des Mantelrandes. $e$ Hinterer Ansatz des Fuß-Retraktors. $f$ Großer, hinterer Schließmuskel-Ansatz. $g$ Muskelansatz der Analmembran. $h$ Freihängendes Periostrakum 
Lage. Vorerst wird das 1. heterochele Schreitbeinpaar, dem Sinnesborsten und damit Chemorezeptoren weitgehend fehlen, nicht eingesetzt, sondern schräg vor dem Körper als mächtiger Schild hergetragen. Die beiden kleinen Chelae der 2. und 3. Schreitbeinpaare treffen median mit weiß abgeschliffenen Chitin-Schneiden aufeinander. Derbe Borstenleisten, die parallel zu diesen Chitin-Schneiden angeordnet sind, erhöhen ihre

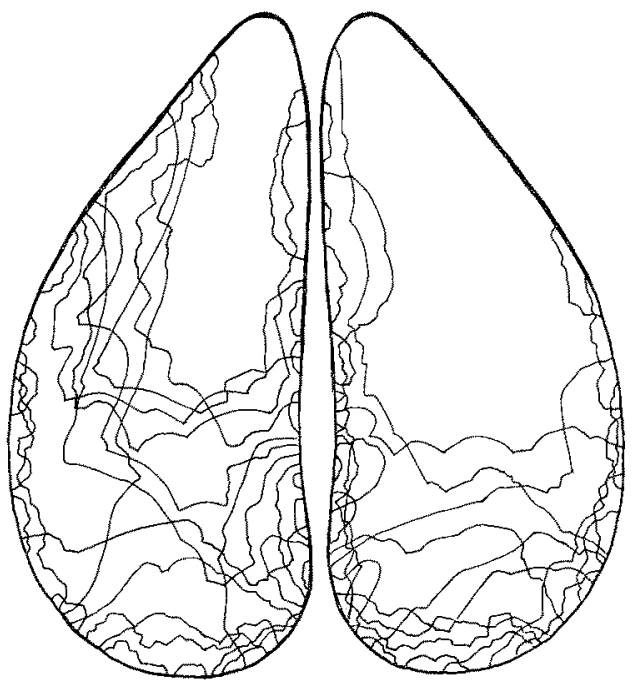

Abb. 4: Kompilatorische Darstellung der an Mytilus edulis beobachteten Nagespuren und Schalenausbrüche. Die jeweils zentral gelegenen Flächen bleiben als Fraßreste erhalten. - Neben einzelnen quer oder diagonal gerichteten Offnungs-Bissen der Makrochela treten kennzeichnend randliche Ausbrüche auf, die beim Abgleiten von Mytilus aus der Knackschere entstehen. Ein Teil der Frakturlinien sind auf das nach dem Freßakt erfolgende Benagen der Schalenreste zurüdkzuführen. (Nad Beobachtungen im Aquarium Münster)

Halte- und Greiffunktion. Von dem 2. und 3. Schreitbeinpaar und den 3. Maxillen wird die Beute nunmehr in die mächtige Knackschere geschoben. Dabei kann das Beutestück wiederholt abgleiten und wird gegen die darübergehaltene, schlanke Greifschere eingespannt, die als zusätzliches Widerlager dient. Durch häufiges Abgleiten hinterlassen die Chelae auf der Muschel charakteristische Kratz-, Schürf- und Nagespuren oder rundliche Randausbrüche. Als Folge davon können das Periostrakum und die blauviolette Prismenschicht der Mytilus-Schalen abgeschliffen werden ebenso wie die obersten Chitinlamellen der großen Chelae (Abb, 1 a und b). Die auf den MytilusSchalen siedelnden Balaniden werden beim Ergreifen bis auf das Basalblatt abgeschürft und gefressen. Der Anteil an Balaniden-Schill übertrift in diesem Fall bei weitem die von Mytilus gelieferten Schalenreste (Beobachtung im Aquarium Münster).

Nun folgt eine langwierige Bewegungsabfolge, die mit einem kräftigen Biß der großen Chela parallel oder senkrecht zur Sagittalebene von Mytilus endet. Er wird quer, schräg oder der Längsachse entsprechend angesetzt, bricht aber oft nur den Außenrand der bereits wieder abrutschenden Beute auf (Abb. 3-7). Nach erneutem, bei Mytilus bisweilen recht umständlichem Einspannen in die Knackschere, gelingt schließ- 
lich der definitive Zubiß. Im folgenden wird der ebenfalls weiß abgeschliffene, sägeblattartige Anhang des Ischio-Basipoditen der 3. Maxillen (Abb. 2 und 8 b), einem wirksamen Halteorgan, weiter die mahlend-kauenden Mandibeln und die mit scharfen Schneiden fest zugreifenden 2. und 3. Schreitbeinpaare zum Fressen der eingeklemmten Beute herangezogen. Raspelnd und nagend wurden - je nach der Größe des

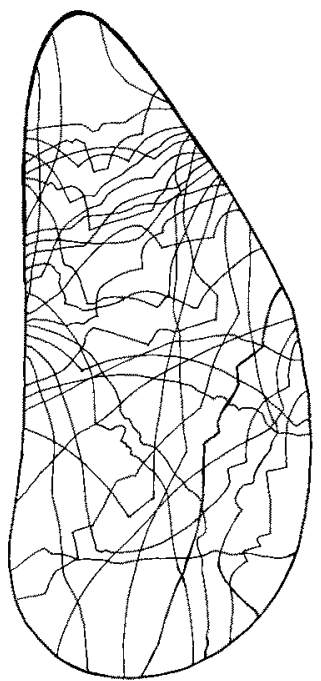

Abb. 5

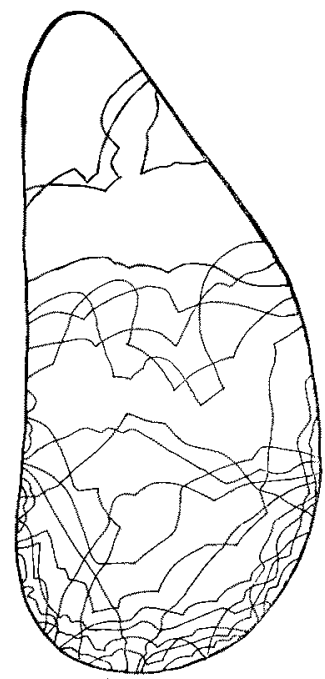

Abb. 6

Abb. 5: Kompilatorische Darstellung von typischen, einmaligen Quer- oder Längsbissen der Knackschere des Hummers, mit denen quer eingespannte Mytilus edulis geöffnet wurden. Kennzeichnend ist bei diesem Versuchstier die Häufung im oberen Drittel der Schale. Längsbrüche treten vergleichsweise zurück. Länge des Versuchstieres Rostrum-Telson ca. $35 \mathrm{~cm}$. (Nach Beobachtungen im Aquarium Helgoland)

Abb. 6: Kompilatorische Darstellung der Frakturlinien an den Fraßresten eines weiteren Tieres. Bei quer in die Knadkschere eingespannten Mytilus edulis überwiegen Schalen-Querbrüche. Die sich häufenden randlichen Ausbrüche sind bei diesem Versuchstier auf das nach dem eigentlichen Freßakt einsetzende Benagen der Schalenreste zurückzuführen. Länge des Versuchstieres Rostrum-Telson ca. $25 \mathrm{~cm}$. (Nach Beobachtungen im Aquarium Helgoland)

Räubers - bei $40 \%$ der beobachteten Mytilus mit den Chelae vom nicht fest abschließenden Innenrand oder vom dünnen, distalen Außenrand her kleine Schalensplitter herausgebrochen (Abb. $1 b-e$ ). Teilweise entstehen dabei auf beiden Klappen symmetrische Ausbrüche (Hollmann 1966, Taf. 47, Fig. 7-8), zumeist sind sie jedoch in kennzeichnender Form auf die Randpartien der Klappen verteilt (Abb. 3-7). Bei über $60 \%$ der Mytilus wurden die linken Klappen stärker zerbrochen als die rechten und deuten damit primäre Härteunterschiede der Klappen an. Bei einigen Muscheln blieb trotz starker Schalenzerstörung noch das Ligament erhalten.

Bei spannlangen Individuen von Homarus werden Muskeln und Bindegewebe jedoch schon aus den nur teilweise aufgebrochenen Klappen herausgezerrt. Die Beute wird dabei von der Makrochela gehalten, oft aber auch zwischen den Chelae des 2. und 3. Schreitbeinpaares eingeklemmt oder fest gegen den Untergrund gestemmt, während die 
Ischiopoditen der 2. und 3. Maxillen die Weichteile zerrupfen. In Brocken gelangen die Bissen über die vorgreifenden, nach unten abgewinkelten Endopoditen der 3. Maxillen, wo die Beute median zwischen Merus, Carpus und Ischium eingeklemmt werden kann, zu den Mandibeln und weiter in den Oesophagus. Of wird die Beute zwischen den Mandibeln gehalten und dann von den Maxillen und Extremitäten herausgezerrt, bis ein Stück abreißt, das verschlungen wird.

Von den zahlreichen, kleinen Muschelsplittern gelangt nur ein geringer Teil auf den Boden, zumeist wandern sie über die Mundgliedmaßen zusammen mit den Nahrungsstïcken in den Schlund und weiter in den Kaumagen. Alle Freßbewegungen der Hummern verschiedener Größen, bei denen die Schale Stück um Stück zerknackt wird, erfolgen ausgesprochen langsam, werden durch mehrfaches Abgleiten der Beute unterbrochen und von lebhaften Bewegungen der Antennen begleitet.

Häufig ist zu beobachten, daß Homarus die von den Fleischresten befreiten Klappen betastet, weiter benagt, und die meisten kleinen Kalksplitter -- wohl zur Deckung

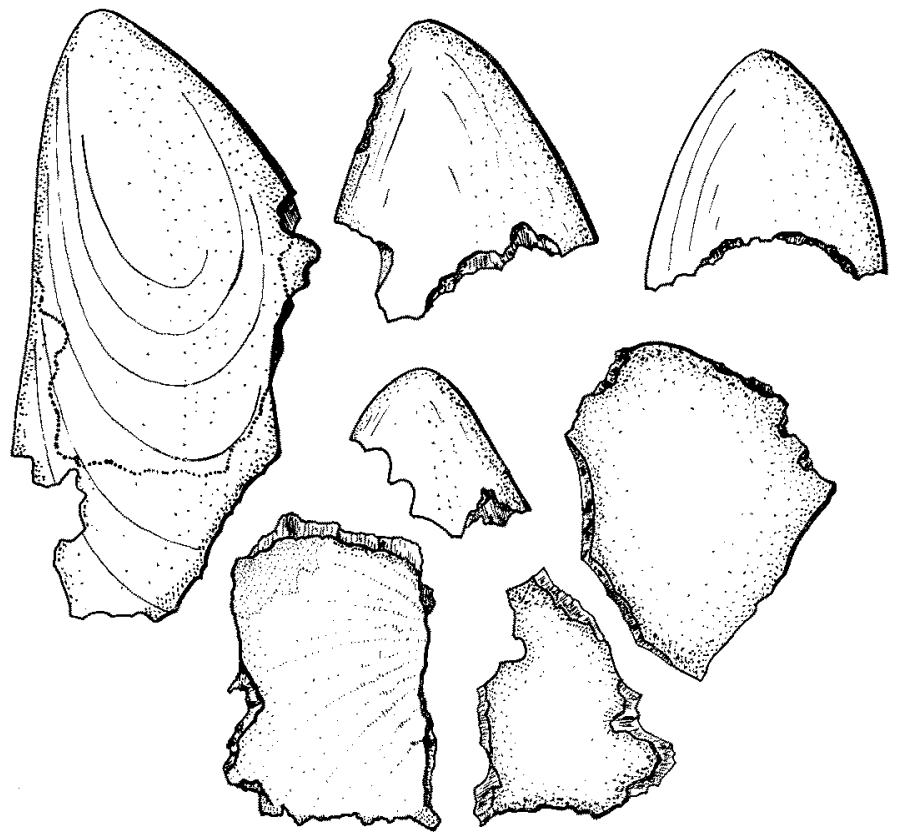

Abb. 7: Fraßreste von Mytilus edulis. Am links dargestellten Exemplar wurde der Verlauf der unteren Bruchlinie punktiert. Die übrigen Schalenscherben sind über das zum Offnen notwendige Maß hinaus zerstört und weiter benagt. (Nach Beobachtungen im Aquarium Münster)

des Kalkhaushaltes (Batss 1926, p. 65, 1927, p. 926) - verschlingt: Als Reste bleiben neben partiell zerstörten Klappen oder deren Teilen insbesondere die stark gewölbte, dickschalige Schloßregion übrig (Abb. 7). Durch dieses Verhalten entstehen oft willkürliche Scherbenformen, die nicht mehr im Funktionskreis des eigentlichen Nahrungserwerbs stehen und vom Freßvorgang unabhängig sind. Sie entziehen sich damit den an 
ein festes Verhaltensschema gebundenen Deutungen, sind in ihren Formen jedoch festgelegt (Abb. $1 d$ und $e$ ).

Schematisch lassen sich an den von Homarus gammarus ïberwältigten Mytilus edulis folgende Schalenzerstörungen erkennen: (1) Am Innenrand, der ungenügend schließt und von lappigem Periostrakum überdeckt ist, in gleicher Weise auch am distalen Außenrand häufen sich Schalenausbrüche (Abb.3-7). Außerdem wird der dünnschalige Außenrand vorzugsweise zur Behebung des Kalkdefizits von Homarus aufgenommen. (2) Uberwiegend werden die Klappen von Mytilus durch ein quer-, diagonaloder längsgerichtetes, einmaliges Zugreifen aufgebrochen. Beim Verzehren der Weichteile wird die Schale oft in weitere, kleinstückige Scherben zerlegt, die meistens mitsamt den Fleischfasern gefressen werden. (3) Aus den Chelae gleitende Muscheln liefern kennzeichnende, bisweilen symmetrisch gestaltete Schalenscherben, deren Kratz-, Schrämm-, Nagespuren und Randausbrüche Rückschlüsse auf den Räuber erlauben. Der nach außen orientierten Funktion der Knackschere entsprechend, liegen die großen Bruchflächen, oftmals ohne das abgesplitterte Ostrakum, auf den Externseiten der Schalenscherben. (4) Nach dem eigentlichen Freßakt kann ein weiteres Benagen und Zerbrechen der Schalen erfolgen, das nicht mehr in das unmittelbare Verhalten der Nahrungsaufnahme gehört. Dabei entstehen extrem deformierte Fraßreste mit einer typischen Folge vieler kleiner Ausbrüche von buchtig-welliger Form (Abb. 1d und $e$, Abb. $4,5,6$ partim, Abb. 7).

\section{Aufbrechen eines Gastropoden, Buccinum undatum}

Anfangs folgt das Appetenzverhalten bei der Nahrungssuche dem gleichen Schema wie bei Mytilus; die Beobachtungen an den verschiedenen Versuchsobjekten stimmen dahingehend überein. Die Sinnesborsten an den 3. Maxillen - speziell an Propodus, Carpus und Merus - sowie an dem 2. und 3, Schreitbeinpaar nehmen die Berührung mit der Beute wahr, die dann vornehmlich mit den 3. Maxillen abgetastet und damit morphologisch eingeordnet werden kann. Anschließend rücken die Extremitäten die Beute zurecht, wenden sie mehrfach, und die Makrochela beginnt - bei bis zu $35 \mathrm{~cm}$ langen Hummern - das Gehäuse vom Außenrand her zu zerstören (Abb. 8a).

So werden zuerst der Siphonal-Stutzen und die angrenzenden Gehäusepartien von Buccinum undatum durch einen kräftigen Zubiß des Hummers abgebrochen. Ebenso wie bei Palinurus vulgaris können vorübergehend „Bandschnitte“ entstehen (SCHÄFER 1962, p. 460), obwohl die Makrochela von Homarus im allgemeinen dafür zu groß erscheint.

Im Regelfall wird jedoch der gesamte Mündungsrand des Buccinum-Gehäuses abgenagt. Auch wird die Schale nach außen herausgebrochen, wobei der Hummer jedoch den größten Teil der abgeraspelten Muschelsplitter verschluckt. Bei der stetigen, nach außen weisenden Aufeinanderfolge der kleinen, randlich angesetzten Ausbrüche ist das Buccinum-Gehäuse ständig in Bewegung und wird um seine Spindel gedreht. Zu diesem Zeitpunkt ist der Gastropoden-Weichkörper bereits extrem kontrahiert. Erst wenn das Schneckengehäuse zu einem "griffigen " Torso deformiert worden ist, reichen die 3. Maxillen und die Chelae der Schreitbeinpaare die Beute der Knackschere zu, die 

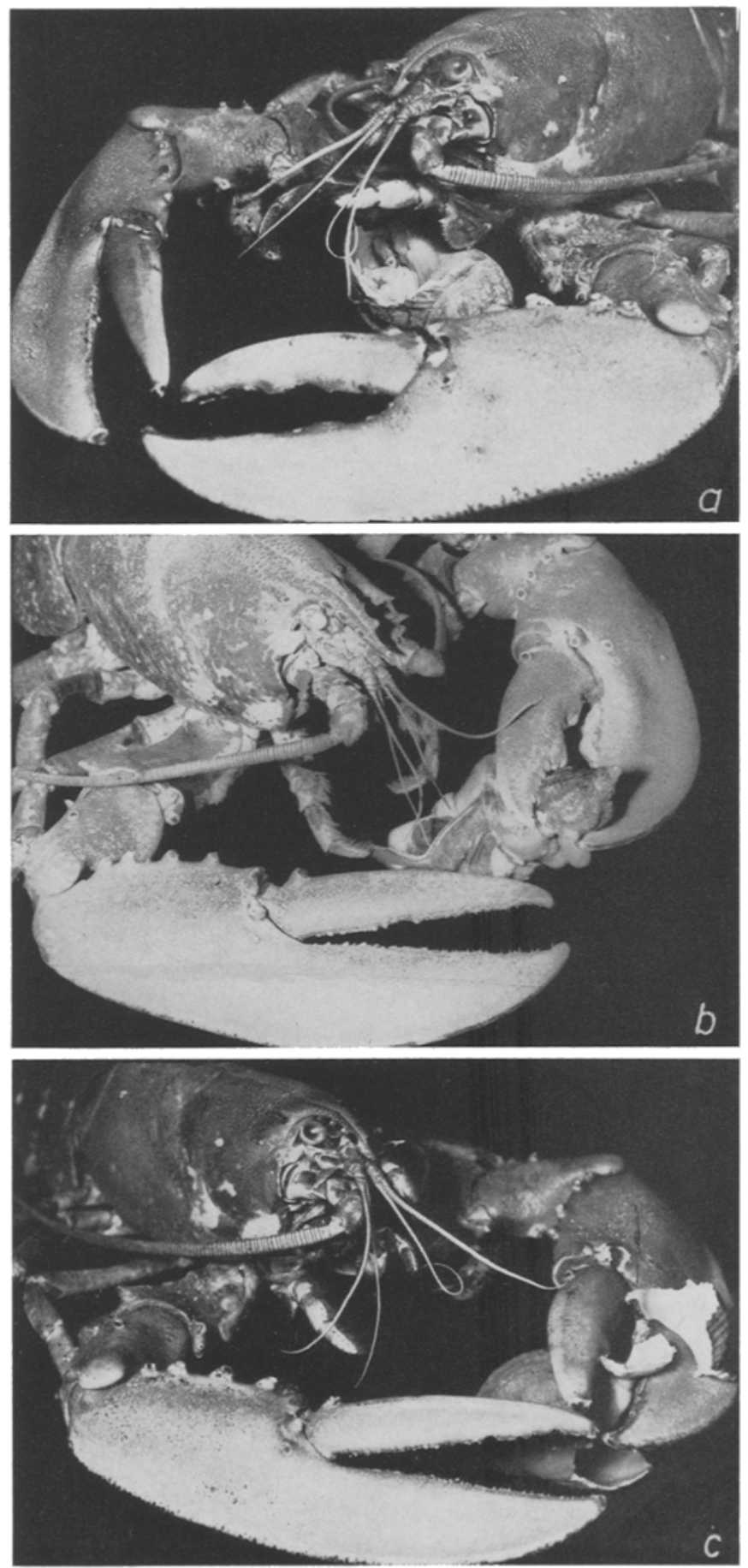
dann den Rest der festen Schale, oft zusammen mit der Spindel, zerbricht (Abb. 8c) oder mit einem Biß den Apex abtrennt (Abb. 8b und 9).

Bei großwüchsigen Hummern mit einer durchschnittlichen Länge Rostrum-Pleuren von 35 bis $40 \mathrm{~cm}$ gilt der erste, oftmals rasche Angriff, der dem orientierenden Betasten folgt, sogleich dem Apex und dem Spindelmuskel. Während die Schnecke noch durch intensivere Bewegungen dem Angreifer zu entgehen sucht und das Gehäuse zudem nur umständlich und nach vielfachen Versuchen in eine für den Zubiß geeignete Lage gerückt werden kann, verstreicht of eine geraume Zeit. Wiederholt gleitet das Gehäuse aus der Makrochela und trägt dabei auffallende, weiße Schrämmspuren davon, die zumeist auf das Ostrakum beschränkt bleiben und die Perlmuttschicht freilegen. Schließlich glïckt der Biß, der den Apex in verschiedener Größe vom Körper trennt ( $\mathrm{Abb}$. 8b und 9). Darauf kann das Gehäuse von zwei Seiten her durch weitere Beißtätigkeit aufgebrochen werden, sowohl von der Spitze als auch von der Mündung ausgehend, bis der Weichkörper für den Zugriff der Mundgliedmaßen freiliegt. Dabei entstehen unförmige Fraßreste von Gastropoden-Gehäusen, denen Apex und Gehäusemündung fehlen (Abb. 9).

Zumeist wird der Torso erneut in die Makrochela eingespannt und gehalten (vgl. Lunz 1947), stützend schiebt sich die Mikrochela davor, die Extremitäten rücken haltend dahinter, und es erfolgt nun der endgültig zerstörende Biß. Dabei kann das Gehäuse in Längs-, Diagonal- oder Querrichtung zerbrechen, und große Teile der Außenumgänge des Gastropoden platzen ab (Abb. 8c). Extremitäten, Maxillen und Mandibeln reißen und zerteilen daraufhin den Weichkörper in kleine Stücke, die erstaunlich schnell im Oesophagus verschwinden.

Als Fraßreste verbleiben große Fragmente der Außenumgänge, die oft kennzeichnende, runde Eindrücke oder Einbrüche der erhabenen Tuberkel auf der Makrochela zeigen. Bisweilen finden sich auch von der Mündung und vom Apex her verstümmelte Gehäusereste oder häufiger isolierte Apices, freigelegte und quergebrochene Spindeln und weniger charakteristische Schalenscherben unterschiedlicher Größe, die nahezu allen Gehäuseregionen zuzuordnen sind. Große Scherben des Außenumganges können auch durch den in das Gehäuse eingreifenden Dactylus und den von außen ansetzenden Propodus herausgebrochen werden (Abb. 10). Viele der Schalenfragmente gehen auf den einmaligen, kräftigen Zugriff der Makrochela und die dabei entstehenden Zerbrechungen des Gehäuses zurück.

Anschließend folgt ein erneutes Betasten, Knacken und Zerraspeln der Schalenscherben, die dabei mehr und mehr ihr typisches Aussehen verlieren. Sie bleiben als willkürlich deformierte Schalenreste erhalten, die keine eindeutigen Rückschlüsse auf den Räuber zulassen. Allerdings scheint die Größe der Scherben, die zumeist eine un-

Abb. 8: a Der Gehäuse-Außenrand eines sich kontrahierenden Buccinum undatum wird von der Makrochela abgeraspelt. Die Gliedmaßen des Endopoditen der 3. Maxille sind deutlich zu erkennen, insbesondere das sägeblattartige Halteorgan an der Innenseiten des Ischio-Basipoditen. $b$ Beispiel für die vorherrschende Reaktionsweise beim Uberwältigen von Buccinum: Der Apex wird durch einen kräftigen Schnitt vom Körper abgetrennt, der die Weichteile freilegt. $c$ Endgültiges Zerbrechen des Gehäuses als Schlußphase: Mit dem Gehäuse-Torso zerbricht die Makrochela of gleichzeitig die Columella und zerlegt die Außenumgänge in große Schalenstiucke. (Aufnahmen: A. Holtmann, Biologische Anstalt Helgoland.) 
mittelbare Beziehung zur Größe des Räubers aufweist, vornehmlich in der Größenordnung von 1 bis $4 \mathrm{~cm}^{2}$ zu liegen, und die nach außen mitsamt dem Ostrakum abgesplitterten Bruchstücke zeigen bei Homarus eine ähnliche Ausbildung wie bei Palinurus. Dadurch unterscheiden sie sich grundsätzlich von anorganisch-mechanisch, durch Bruch gebildeten Schalenschillen.

Eigenartigerweise werden auch leere Gehäuse von Buccinum abgetastet, benagt und schließlich in der dargestellten Reihenfolge in Bruchstücke zerlegt. Als Vorstufen
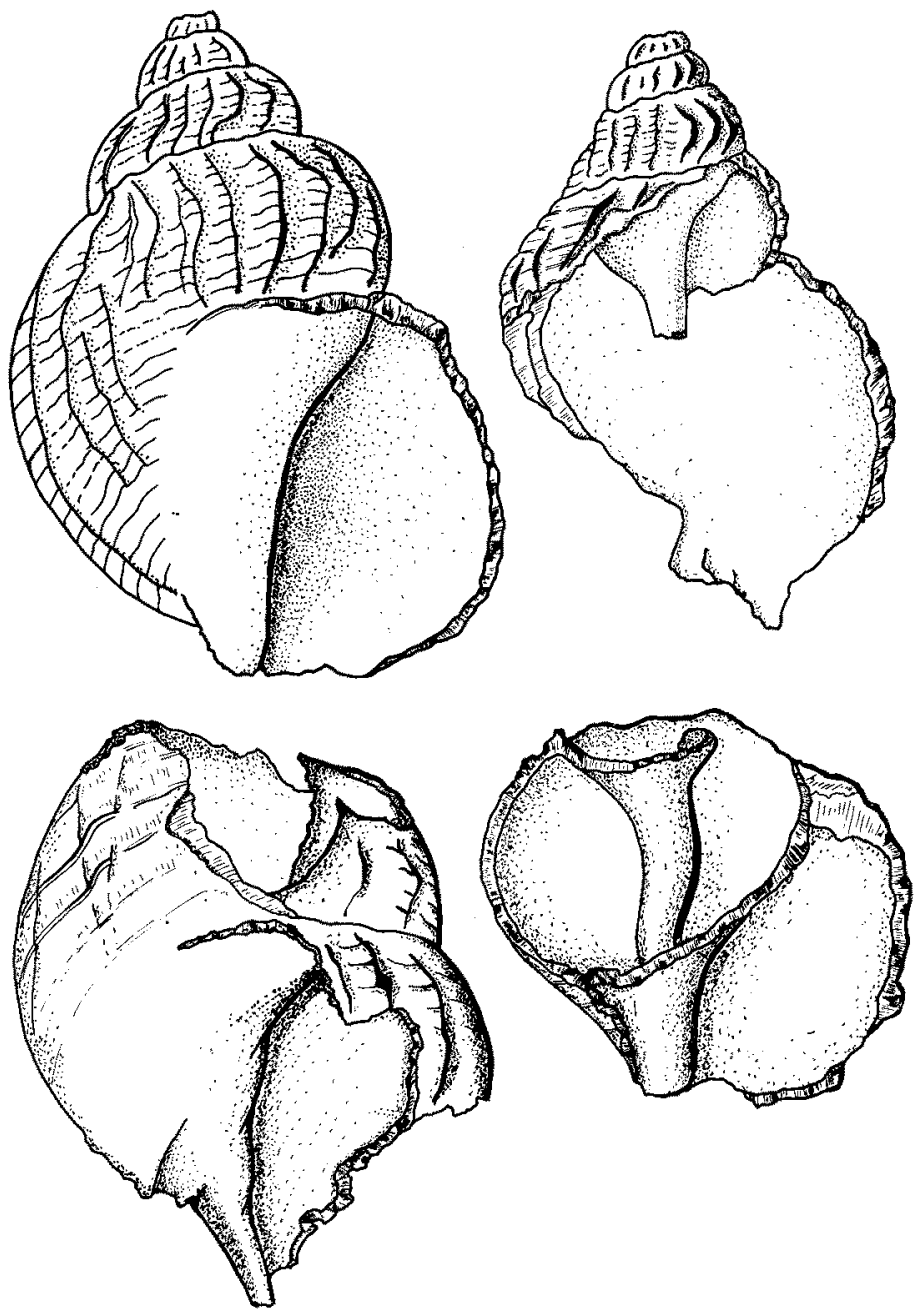

Abb. 9: Fraßreste von Buccinum undatum. Links oben: Anfangsstadium der Zerstörung des Außenrandes und des Siphonal-Stutzens mit deutlichen Raspel-Spuren. Links unten: Ein dem Hummer vorzeitig abgenommenes Beutestück, dem der Apex bereits fehlt. Das Gehäuse wurde von der Spitze und von der Offnung her verschieden stark angegriffen. Rechts: Endphasen der Gehäuse-Zerbrechung; die Weichteile der Schnecken wurden bereits gefressen. (Material: A. Holtmann, BAH) 
der endgültigen Zerstörung oder bei Unterbrechungen des Freßaktes können hierbei die typischen „Bandschnitte“ entstehen (PAPP et al. 1947, p. 291).

Auch für Buccinum ergibt sich eine stufenweise Folge der Schalenzerstörung: (1) Großwüchsige Hummern brechen zuerst den Apex von Buccinum ab. Der entstandene Torso kann von oben und von der Mündung her weiter aufgeknackt werden. Der Restkörper wird schließlich durch einen kräftigen $\mathrm{Bi}$ in relativ grobe Schalenscherben zerlegt. (2) Kleinwüchsige Hummern greifen anfangs auch nach dem Apex, benagen und brechen große Buccinum dann jedoch von der Mündung her auf. Als Fraßreste bleiben vergleichsweise kleinstückige Reste des Apex, der Columella und einfache Schalenscherben. (3) In diesem besonderen Fall kommt dem Größenverhältnis Räuber Beute eine entscheidende Rolle zu.
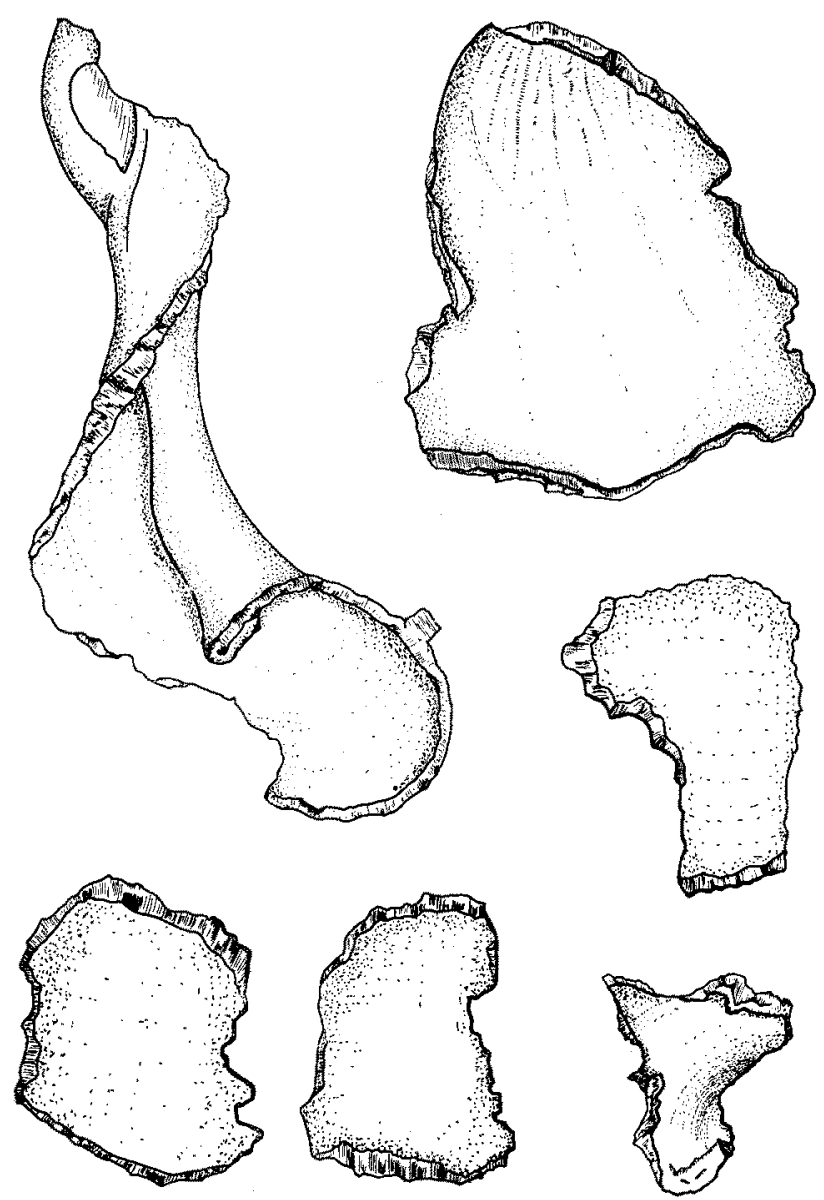

Abb. 10: Letzte Fraßreste der Gehäuse von Buccinum undatum; die freigelegte Columella und willkürlich herausgebrochene Stidke der Außenumgänge. (Material: A. HoLTMAnN, BAH) 


\section{DIKUSSION}

Die oben wiedergegebenen Beobachtungen, die teils im Aquarium Münster, teils im Aquarium der Biologischen Anstalt Helgoland gesammelt wurden, zeigen - abweichend von den Untersuchungen von SHoup (1968) an Vertretern der Gattung Calappa - daß auch bei Homarus gammarus ein weitgehend spezialisiertes Verhalten beim Aufbrechen von Mollusken vorliegt. Im übrigen stellen die Mollusken nur einen Ausschnitt aus dem Beutespektrum von Homarus dar, so daß weitere Untersuchungen, die andersartige Reaktionsfolgen erkennen lassen könnten, notwendig sind.

Der zeitliche Ablauf vom ersten Ergreifen der Beute bis zu deren endgültigem Verzehr schwankt erheblich; Verzögerungen ergeben sich insbesondere durch das Abgleiten nicht "griffiger" Bentestücke sowie durch die in vielen Fällen ausgesprochen langsamen Bewegungen beim Zerknacken der Schalen. Zum orientierenden Erfassen beschalter Beutestücke werden wechselweise die 3. Maxillen (mit Sägen bewehrte Halteorgane!), die Mandibeln sowie das 2. und 3. Schreitbeinpaar eingesetzt. Ubereinstimmend werden Beutestücke mit festen Körperhüllen von der Makrochela zerbrochen, wie es bereits HAGMEIER \& KüNNE (1950, p. 193) beobachteten. Auch die gesamte Kleinarbeit beim Zerstören von Schalen und Gehäusen wird von der Knackschere geleistet, deren Bewegungen von den anderen Extremitäten wirkungsvoll unterstützt werden. Dabei entstehende Schalenscherben können - dem Aktionsradius des Räubers entsprechend - über ein weite Fläche verstreut werden.

Unterbrechungen beim Freßakt, die besonders deformierte Scherbenformen liefern könnten, wurden in keinem Fall beobachtet. Beim zielstrebigen Nahrungsverhalten folgten dem Angriff auch stets die vollständige Überwältigung der Beute. Willkürliche Störungen und Eingriffe in den Freßakt mit dem Ziel, den Fortschritt der Schalenzerbrechung zu beobachten, irritierten die Versuchstiere bei der Nahrungsaufnahme nicht in nennenswerter Weise.

Einmal beeinflußt die Menge des Nahrungsangebotes die Größe der Scherbenreste: Das Beutestïck wird in einem relativ kurzen Akt partiell aufgebrochen, und die am leichtesten zugänglichen Weichteile werden verzehrt. Als Ergebnis dieses einfachen Aufbrechens entstehen große Schalenscherben mit relativ glatten Bruchflächen, die nur einen begrenzten Bereich überstreuen. Obwohl einzelnen Schalenfragmenten noch Fleischfasern anhaften, wendet sich der Krebs bereits einem neuen Beutestück zu.

Bei einzelnen, den Versuchstieren gereichten Nahrungsstücken zieht sich der gesamte Freßvorgang oftmals über Stunden hin. Die bereits zerkleinerten Schalen von Mytilus und auch Buccinum werden mehrmals erneut aufgenommen, ausgiebig betastet und mit den Mandibeln bearbeitet. Anschließend werden sie umständlich in die Makrochela geschoben und weiter zerknackt. Als Ergebnis solcher langfristigen Bearbeitung liegen dann kleine Schalenreste in einem weiten Bereich verstreut, soweit sie nicht verschlungen wurden.

Das Größenverhältnis Räuber-Beute spielt bei diesen Vorgängen eine recht variable Rolle: Was einem großen Hummer in relativ kurzer Zeit gelingen mag, erreichen kleinwüchsige Artvertreter in einem entsprechend längeren Zeitraum. Die Scherbengrößen der Fraßreste und ihre Formen geben daher über den Hergang des Freßaktes nur bedingt Aufschluß; grobe Schalenbruchstücke sprechen für rasch über- 
wältigte Beutestücke. Kleine Schalenscherben können hingegen sowohl als Fraßreste eines kleinen Räubers wie auch als Ergebnis der intensiven Beschäftigung eines großen Räubers mit seiner Beute angesehen werden. Kennzeichnend treten dann an den dünnen Schalenpartien wellig-buchtige Ausbrüche auf.

Bewegliche Beutetiere, etwa Krabben oder auch Gastropoden, werden mit einer raschen Bewegung der Greifschere erfaßt und mit Hilfe der übrigen Extremitäten in die Knackschere geschoben. Vom Berühren und Erfassen der Beuteobjekte über das Zerbrechen der festen Außenhüllen bis zum eigentlichen Freßakt ergibt sich eine Vielfalt einzelner Reaktionsschritte. Welcher davon im jeweiligen Fall zur Ausführung gelangt, ist vom Größen- und Art-Verhältnis Räuber-Beute, dem Nahrungsangebot sowie von der Plastizität im Verhaltensschema des Räubers abhängig.

Allem Anschein nach ist in der gesamten Lebensweise und damit auch im Verhalten großwüchsiger Hummern ein weiterer Gesichtspunkt zu berücksichtigen. Das zum Beuteerwerb ebenso wie zur Abwehr umgeformte 1. Schreitbeinpaar entwickelt sich während des ontogenetischen Wachstums anscheinend allometrisch. Die dem juvenilen Tier primär überaus wichtigen Greif-, Reiß- und Abwehrorgane überschreiten bei Exemplaren, die $40 \mathrm{~cm}$ Körperlänge erreicht haben, ihre funktionellen Aufgaben, die in zunehmendem Maße - jedenfalls in der Gefangenschaft - von den 3. Maxillen, speziell aber vom 2. und 3. Schreitbeinpaar übernommen werden. Makro- und Mikrochela hingegen wirken beim Nahrungserwerb und bei den Abwehrreaktionen als allometrisch-überdimensionierte Organe, die über ihre ursprünglichen Aufgaben hinausgewachsen sind und im Kampf ums Dasein eher Nachteile als Vorteile mit sich bringen.

\section{ZUSAMMENFASSUNG}

1. Dem Beuteverhalten von Homarus gammarus (L.) gegenüber Mytilus edulis (L.), einem Lamellibranchiaten, sowie gegenüber Buccinum undatum L., dem im Biotop typisch vergesellschafteten Gastropoden, wurde anhand von Aquariums-Beobachtungen nachgegangen.

2. Beide dieser Beute-Objekte aus dem weitgespannten Beutespektrum von Homarus, zu dem Echinodermen, Crustaceen und eine Vielzahl anderer Benthonten des Litorals gehören, klären die Entstehung charakteristischer, fossilisationsfähiger SchalenFraßreste. Deren Morphologie wird dargestellt und ihre Entstehung besprochen.

3. Im anpassungsfähig-plastischen Verhalten dieses und zahlreicher anderer Dekapoden bleiben darüber hinaus vielfältige morphologische Freiheitsgrade, die in der Ausgestaltung und Formgebung von Mollusken-Bruchscherben weitere Möglichkeiten offenlassen. Jedoch zeigt sich auch eine gewisse Einengung durch gemeinhin festliegende Reaktionsfolgen der überwiegend im Litoral beheimateten Dekapoden.

Danksagungen: Herrn Prof. Dr. R. Altevogr, Münster, sowie Herm Prof. Dr. H. Hölder, Münster, danke ich für wichtige Hinweise. Mein besonderer Dank gilt Herrn A. Holtmann, Leiter des Aquariums der Biologischen Anstalt Helgoland, der mit seinen reichen Erfahrungen bei der Hummern-Aufzucht wie auch durch typisches Material und Photographien die vorliegenden Untersuchungen wesentlich unterstützte. Mein Dank gilt ebenfalls Herm Dr. 
H. Rrichlnve und Herrn U. KonnerT, Zoologischer Garten Münster, die zahlreiche Aquariums-Versuche möglich machten. Herrn cand. rer. nat. P. JATZKe, Biologische Anstalt Helgoland, danke ich für anregende Diskussionen sowie für die Möglichkeit zusätzlicher AquariumsBeobachtungen.

\section{ZITIERTE LITERATUR}

Altevogt, R., 1963. Lernversuche bei Uca tangeri. Zool. Beitr. (N.F.) 9, 447-459.

Balss, H., 1926. Decapoda. Tierwelt N.- $u$. Ostsee 10 h 2, 9-112; h 3, 1-5.

- 1926/1927. Crustacea malacostraca. 14. Ordnung: Decapoda LATREILLE 1802. Handb. Zool. 3, 840-1038.

- Korschelt, E., Buddenbrock, W. von \& Gruner, H. E., 1940-61. Decapoda, Bronn's Kl. Ordn. Tierreichs 5 (Abt. 1, Buch 7), 1-2169.

Broch, F., 1926. Das Verhalten des Einsiedlerkrebses Pagurus arrosor Herbst während der Suche und Aufnahme der Nahrung. Z. Morph. Okol. Tiere 6, 415-552.

CADÉE, G. C., 1968. Molluscan biocoenoses and thanatocoenoses in the Ria de Arosa, Galicia, Spain. Proefschrift. Rijksuniversiteit, Leiden, 121 pp.

CARTER, R. M., 1968. On the biology and palaeontology of some predators of bivalved mollusca. Palaeogeogr. Palaeoclimat. Palaeoecol. 4, 29-65.

Hagmeier, A. \& Künne, C., 1951. Die Nahrung der Meerestiere. Handb. Seefisch. Nordeur. 1 (5b), 87-242.

Herrick, F. H., 1896. The american lobster: A study of its habits and development. Bull. U.S. Fish Commn 15 (1895), 1-252.

Hollmann, R., 1968. Zur Morphologie rezenter Mollusken-Bruchschille. Paläont. Z. 42, $217-235$.

- 1966. Tauchbeobachtungen zur Mollusken-Verbreitung und Sediment-Umlagerung im Golf von Neapel. Neues Jb. Geol. Paläont. Abh. 115, 499-526.

KREJCI-GraF, K., 1926. Schalenanhäufungen und Einbettungslage im Pliocän. Senckenbergiana $8,6-15$.

Lunz, G. R., 1947. Callinectes versus Ostrea. J. Elisha Mitchell scient. Soc. 63, 81.

Papp, A., Zapfe, H., Bachmayer, F. \& Tauber, A. F., 1947. Lebensspuren mariner Krebse. Sber. Akad. Wiss. Wien (Math.-nat. Kl. Abt. 1) 155, 281-317.

SснÄғвR, A., 1954. Form und Funktion der Brachyuren-Schere. Abb. senckenb. naturforsch. Ges. 489, 1-65.

Schäfer, W., 1962. Aktuo-Paläontologie nach Studien in der Nordsee. Kramer, Frankfurt a. M., $666 \mathrm{pp}$.

Schmiтr, W. L., 1965. Crustaceans. Univ. of Michigan Press, Ann Arbor, 204 pp.

SсHÖNE, H., 1961. Complex behaviour. In: The physiology of crustacea. Ed. by T. H. Waterman. Academic Press, New York, 2, 465-520.

SHoup, J. B., 1968. Shell opening by crabs of the genus Calappa. Science, N. Y. 160, 887-888.

Teichert, C. \& Serventy, D. L., 1947. Deposits of shells transported by birds. Am. J. Sci. 245, 322-328.

Walther, J., 1910. Die Sedimente der Taubenbank im Golf von Neapel. Abh. preuss. Akad. Wiss. (Math.-nat. Kl.) 1910, 49.

WILson, J. B., 1967. Palaeoecological Studies on shell-beds and associated sediments in the Solway Firth. Scott. J. Geol. 3, 329-371.

Yonge, C. M., 1960. Oysters. Collins, London, $209 \mathrm{pp}$.

Anschrift des Autors: Dr. R. HollmanN

Geologisch-Paläontologisches Institut

der Universität Münster

44 Münster (Westf.)

Pferdegasse 3 